\title{
A Review on Microwave Based Moisture Measurement System for Granular Materials
}

\author{
Twisha Pandey ${ }^{1}$, Tapas K Bhuiya ${ }^{2}$, Ravi Singh ${ }^{3}$, Bharti Singh ${ }^{4}$, Rajesh Harsh ${ }^{5}$ \\ ${ }_{1,4}$ (EXTC Department, K.J. Somaiya CoE, University of Mumbai, India) \\ 2,3,5 (SAMEER, IIT Campus, Powai, India)
}

\begin{abstract}
In the recent years, development of a non-destructive and non-contacting type moisture measurement system has attracted considerable interest. Because of their inherent nature of being nondestructive, ability to travel through non-conductive medium and having deep penetrative properties, Microwaves have been the traditional choice in this kind of research. This paper is a comprehensive review of Microwave Based Measurement System used for grains and pulses. In this paper, moisture measurement and calibration techniques for the system are presented. The objective of the paper is to provide the readers a broad perspective of the various techniques.
\end{abstract}

Keywords - Bulk density, Complex Permittivity, Moisture Measurement, Temperature Compensation

\section{INTRODUCTION}

The moisture content of materials determines their suitability for storage, commerce and industrial processing. If the moisture content of grains is too high, they are deemed unfit for storage and must be dried to avoid decay. Traditionally, oven drying and weighing of samples was done for moisture measurement. In [1], a well defined protocol specifies weighing the sample initially. The sample is then oven dried and weighed again. The difference in weights divided by the initial weight gives the moisture content. However, in the drying process, not only water but also other volatile substances get evaporated leading to erroneous results. Another method conventionally used was the Karl Fischer test. This method is destructive and time consuming.

The concept of using microwaves for moisture measurement evolved out of the need to overcome the above limitations. Moisture measurement using microwaves is real time, non-destructive and more accurate. However this method also suffers from errors resulting due to bulk density and temperature variations. Both these factors have to be either eliminated or compensated during calibration.

\section{PROPERTIES OF MOISTURE (WATER)}

Water has a bipolar structure i.e. while water molecules are neutral as a whole; one end of the water molecule tends to have a positive charge while the other has a negative charge. In such molecules, the electric field of microwaves causes oscillations. These oscillations lead to loss due to absorption and reflection of energy. Higher the moisture content, greater will be the loss.

Distilled water has a high dielectric constant of about 80.10 at $20^{\circ} \mathrm{C}$ due to the dipole moment and can be polarized. The electrical properties (dielectric property and loss tangent) of any material also depend on the frequency of operation. Complex permittivity is given as:

$$
\varepsilon(\mathrm{w})=\varepsilon^{\prime}(\mathrm{w})-\mathrm{j} \varepsilon^{\prime \prime}(\mathrm{w})
$$

Here, $\varepsilon^{\prime}(w)$ is the real part of permittivity and represents the stored energy within the material and $\varepsilon^{\prime \prime}(\mathrm{w})$ is the imaginary part of permittivity and represents dissipation of energy within the medium. The negative sign of the imaginary part denotes loss of energy [2].

\section{MEASURING PHASE SHIFT AND ATTENUATION}

The sample under test i.e. the sample for which moisture content has to be determined has to be first prepared. This sample preparation involves spraying distilled water on the grains, storing them in sealed jars for 72-96 hours. The samples are mixed periodically by rotating the sealed jars thus ensuring uniform moisture throughout the sample [3].

The sample is then placed in a sample holder of uniform thickness. Horn antenna is used to transmit microwaves into the sample. The transmitted waves undergo reflection and absorption due to the moisture in the sample and are received by another horn antenna at the other end of the sample holder as shown in the figure. Microstrip patch antennas can also be used instead of horn antennas leading to light weight system as reported in [5]. However, horn antennas offer the advantages of high gain and high directivity as compared to microstrip patch antennas which is crucial in for accurate measurements [4]. 


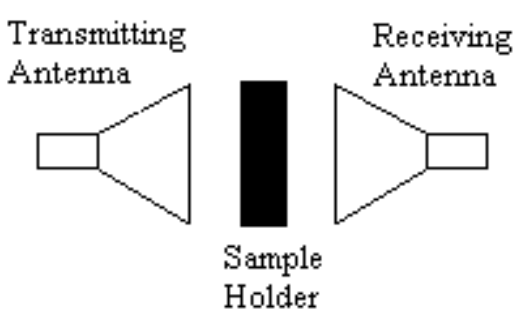

Fig. 1. Schematic diagram showing horn antennas and sample holder for moisture measurement

Attenuation and phase shift are determined by comparing the reference microwave signals measured without the sample and those obtained after the sample is placed between the two antennas. Attenuation is the difference in power levels without the sample $\left(\mathrm{P}_{\mathrm{RF}},{ }_{0}\right)$ and with the sample $\left(\mathrm{P}_{\mathrm{RF}}, \mathrm{s}\right)$ placed between the transmitting and receiving antennas [4].

$$
\Delta \mathrm{A}(\mathrm{dB})=\mathrm{P}_{\mathrm{RF}, \mathrm{s}}(\mathrm{dB})-\mathrm{P}_{\mathrm{RF}, 0}(\mathrm{~dB})
$$

Phase shift is characterized by slowing of the waves in the medium. It is given by phase measured without the sample $\left(\Phi_{0}\right)$ and phase measured with the sample $\left(\Phi_{\mathrm{s}}\right)[4]$.

$$
\Delta \Phi(\mathrm{rad} / \mathrm{m})=\Phi_{\mathrm{s}}-\Phi_{0}
$$

\section{CALCULATION OF COMPLEX PERMITTIVITY}

From $\Delta \mathrm{A}$ and $\Delta \Phi$ as calculated in (2) and (3), the real and imaginary parts of permittivity have to be determined. The steps for this calculation are as follows [6]:

$$
\begin{aligned}
& \alpha=\frac{\Delta A}{8.686 d} \\
& \beta=\frac{\Delta \Phi}{d}+\beta o \\
& \beta o=2 \pi \beta 0 \\
& \varepsilon^{n}=\left(\frac{\beta}{\beta o}\right)^{2} \\
& e^{x}=\frac{2 \alpha \beta}{\beta o}
\end{aligned}
$$

From (6) and (7) the values of real and imaginary part of permittivity are calculated. Thus the complex permittivity is determined.

\section{CALIBRATION FOR DETERMINING MOISTURE CONTENT}

A graph of $\Delta \mathrm{A}$ and $\Delta \Phi$ plotted against the moisture content shows an approximately linear relationship as shown in Fig. 2 and Fig. 3 [7].

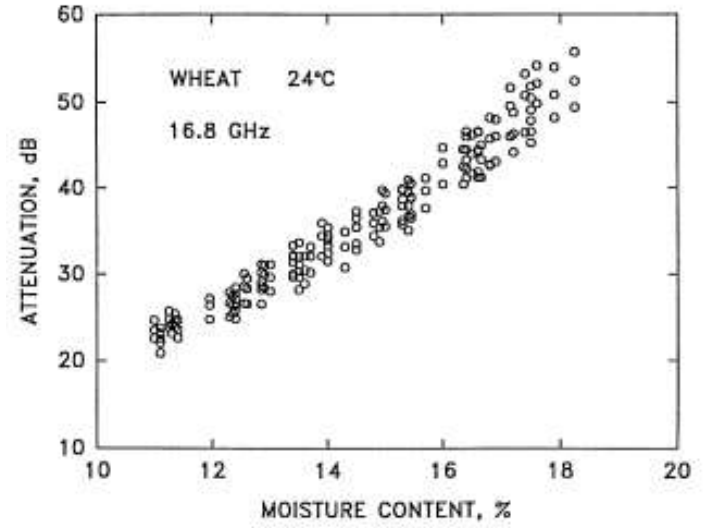

Fig. 2. Attenuation vs. Moisture Content [7]

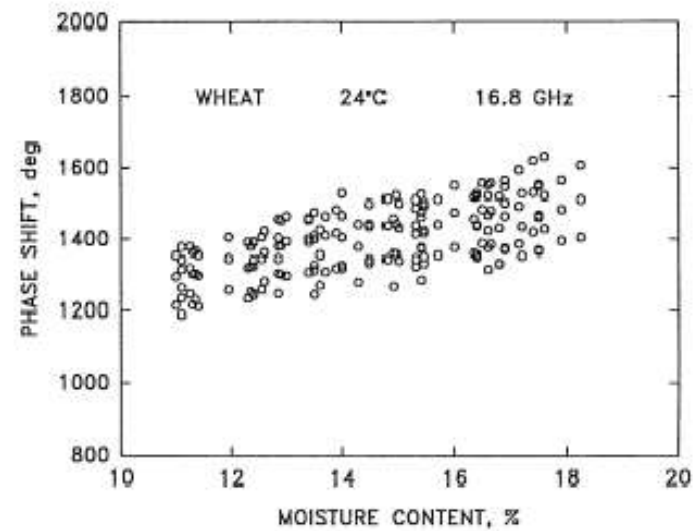

Fig. 3. Phase Shift vs. Moisture Content [7]

In [8] a third order polynomial regression model was proposed to describe the relation between receiving antenna measurements and the moisture content. If the moisture content is denoted by $\mathrm{MC}, \mathrm{V}$ is the 
A Review on Microwave Based Moisture Measurement System for Granular Materials

output of the voltage detector connected to the receiving antenna and $\rho$ is the bulk density in $\mathrm{g} / \mathrm{cm}^{3}$, then the polynomial for determining the moisture content is given by:

$M C=-1.0896 \times 10^{-9} V^{1}+6.4620 \times 10^{-6} V^{2}+1.307 \times 10^{-1} V+4.6 \times 10^{-4} T+0.1845 \times \frac{100}{\rho}$

However, as can be seen, the moisture content varies with the bulk density and this has to be compensated while developing the regression model. There was a need to develop models which were independent of bulk density. The ratio method was then developed as reported in [7]. The method involved using a function which was ratio (R) of the two parameters $\Delta \mathrm{A}$ and $\Delta \Phi$. Since, both $\Delta \mathrm{A}$ and $\Delta \Phi$ depend on bulk density (Fig. 4), their ratio, $R$ is essentially independent of bulk density (Fig. 5).

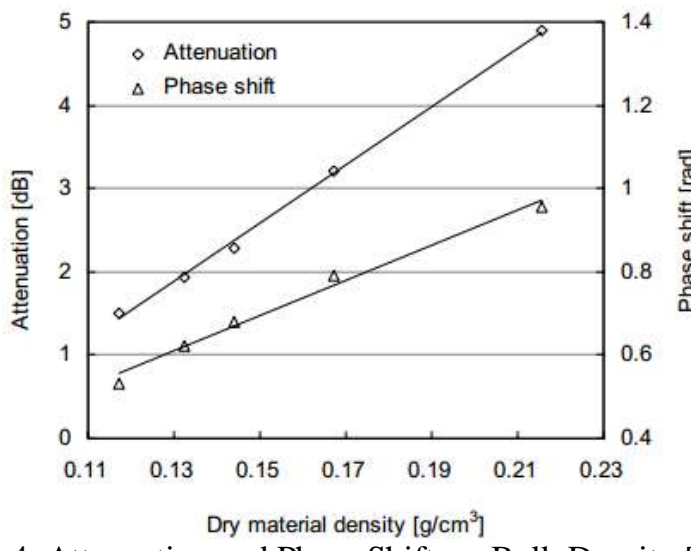

Fig. 4. Attenuation and Phase Shift vs. Bulk Density [9]

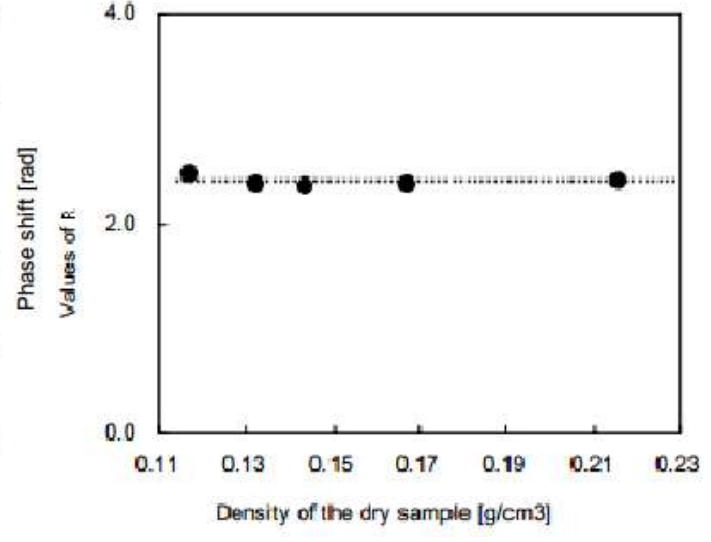

Fig. 5. Ratio R vs. Bulk Density [9]

$$
R=\frac{\Delta A}{\Delta \Phi}
$$

A linear polynomial regression model was proposed specifying the calibration equation for moisture content in terms of $R$. The equation also considered the dependence of $R$ on temperature (T) [7].

$$
M C=\frac{\left(10^{\mathrm{a}} R+12.768\right)-0.1438 T}{0.01185 T+2.1003}
$$

However, this method suffers from decreased accuracy for very dry and very moist materials. This two parameter method is more suitable for transmission lines. It also does not calculate bulk density of the sample [3]. A new density independent, temperature compensated calibration function was proposed in [3]. In this method, complex permittivity is used for calibration. The real and imaginary parts of permittivity are calculated using (6) and (7). A plot of $\varepsilon$ " vs. $\varepsilon$ ' is obtained as shown in Fig. 6.

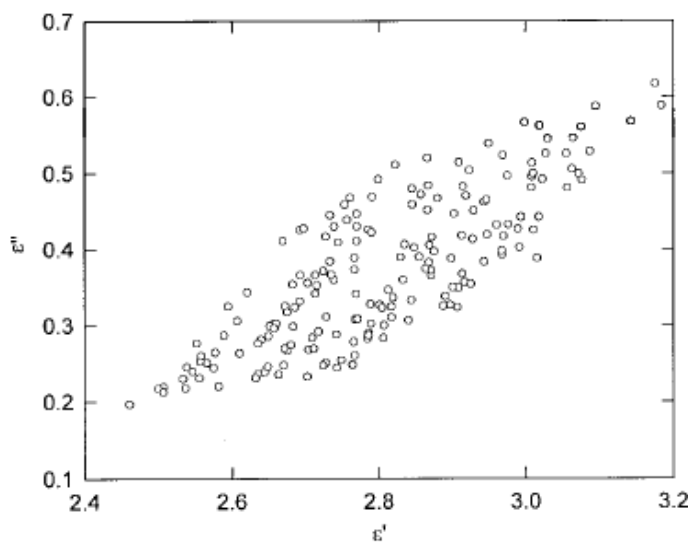

Fig. 6. Imaginary part vs. Real part of Complex permittivity [3]

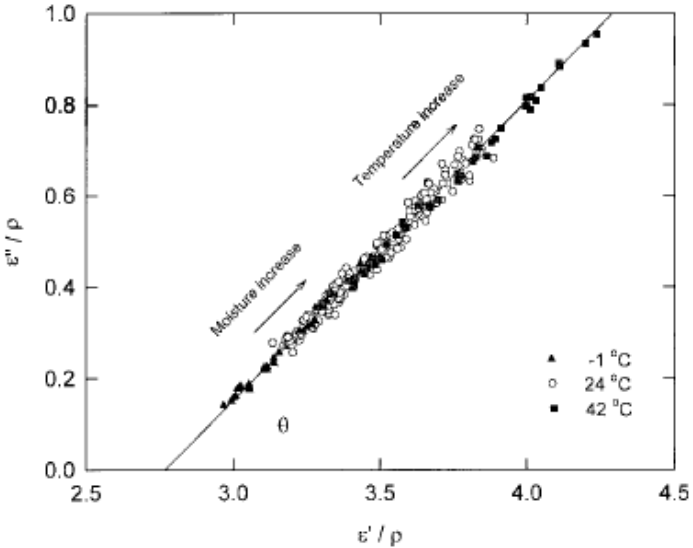

Fig. 7. Imaginary part vs. Real part of Complex Permittivity normalized by bulk density [3]

The data points are obtained as a cluster. This shows the dependence of complex $\varepsilon$ ' and $\varepsilon$ " on bulk density. However, if $\varepsilon$ ' and $\varepsilon$ "' are normalized by the bulk density as shown in Fig. 7, the bulk density effect is reduced and instead of a cluster of points, we obtain an approximate linear relationship. Thus calibration function obtained from such a plot will be bulk density independent. 
A Review on Microwave Based Moisture Measurement System for Granular Materials

The slope of this plot is denoted by $\mathrm{m}$ and the $\mathrm{x}$ intercept is denoted by $\mathrm{k}$. Thus we have [3]:

$$
\frac{\varepsilon^{\mathrm{m}}}{\rho}=\mathrm{m}\left(\frac{e^{\mathrm{e}}}{\rho}-\mathrm{k}\right)
$$

A separate density measuring instrument incurs extra cost, increases the technical complexity in installation of the measuring system. This problem is alleviated using this calibration technique and the bulk density of the material can be calculated from (11) as:

$$
\rho=\frac{1}{\mathbb{k}} \frac{m \varepsilon^{s}-\varepsilon^{n}}{m}
$$

Measurements carried out at various frequencies in [3] showed that the value of $\mathrm{k}$ remains nearly constant at 2.76 and $\mathrm{m}$ varies with frequency according to the following equation [3]:

$$
m=0.0184 f+0.3826
$$

The loss tangent $\tan \delta$ which is a ratio of $\varepsilon "$ and $\varepsilon$ ' increases with increase in moisture content. However, bulk density variations also affect $\tan \delta$ and a cluster of data points is obtained (Fig. 8). To reduce this effect, a ratio of $\tan \delta$ and bulk density obtained in (12) can be taken. Let this ratio be denoted by $\Psi$ [3]:

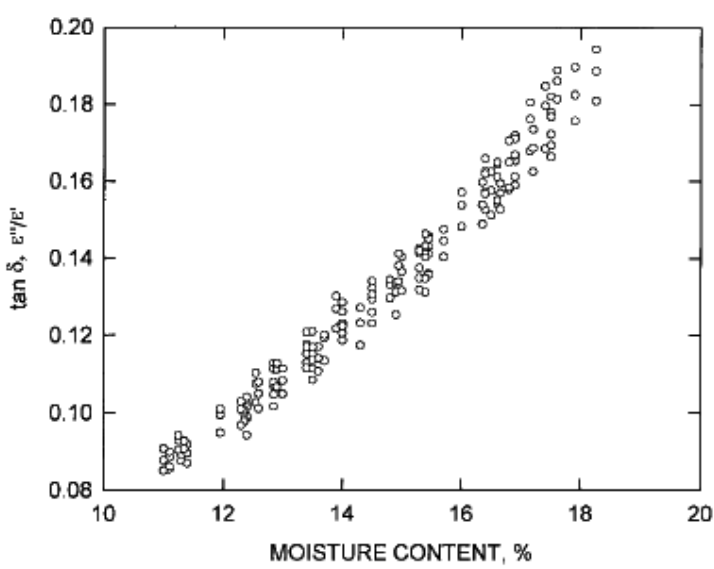

Fig. 8. Loss tangent vs. Moisture Content [3]

$$
\Psi=\frac{k m}{m \varepsilon^{p}-\varepsilon^{m}}\left(\frac{\varepsilon^{m}}{\varepsilon^{p}}\right)
$$

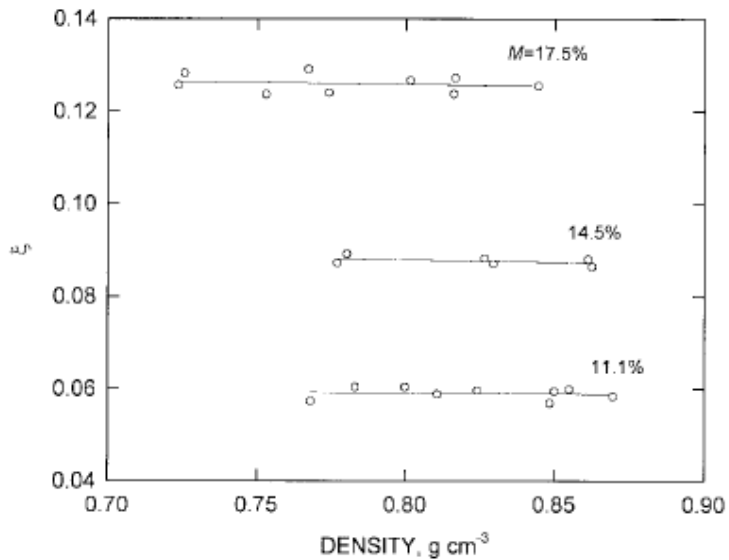

Fig. 9. Density Independent Function vs. Density [3]

At a given frequency, the product of $\mathrm{k}$ and $\mathrm{m}$ is a constant and can be omitted. So, at a single frequency, the density independent calibration function is defined as [3]:

$$
\xi=\frac{\varepsilon^{m}}{\varepsilon^{t}\left(m \varepsilon^{r}-\varepsilon^{m}\right)}
$$

$\xi$ is independent of density (Fig. 9). The square root of $\xi$ varies linearly with moisture content (Fig. 10). Thus linear regression model can be developed showing the relationship between $\xi^{1 / 2}$ and moisture content.

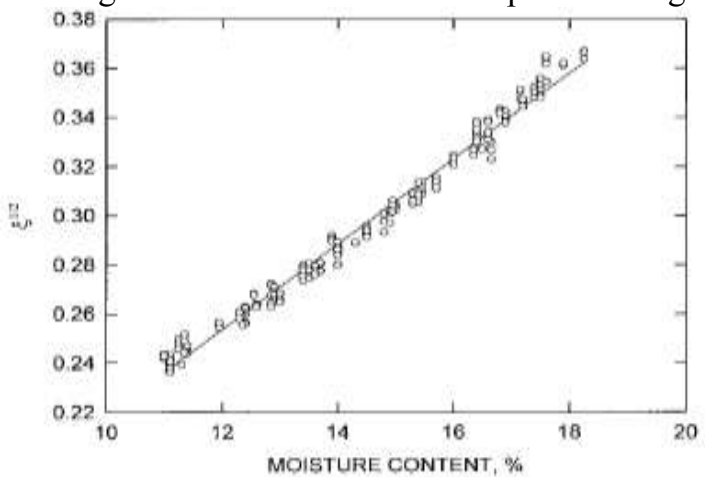

Fig. 10. Square root of Density Independent Calibration Function vs. Moisture Content [3]

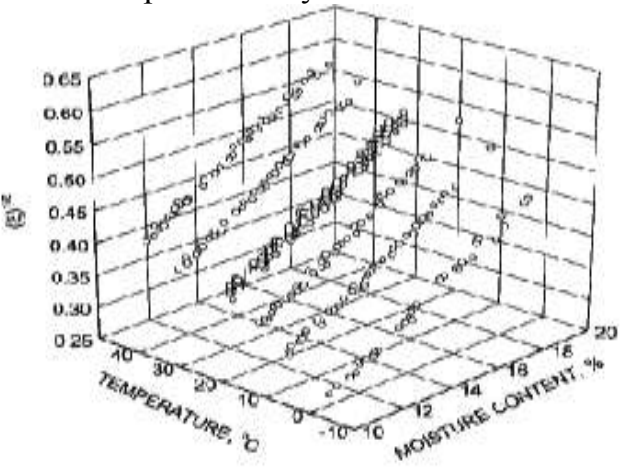

Fig. 11. Temperature Dependence of Square root of Density Independent Calibration Function [3]

$$
\sqrt{\xi}=A(M C)+B
$$


In this linear regression to fit the data, $\mathrm{A}$ is the slope, $\mathrm{MC}$ is the moisture content and $\mathrm{B}$ is the $\mathrm{x}$ intercept. In [3], it is reported that the value of A remains constant with temperature; however B is a function of temperature. Again performing regression, the value of $B(T)$ where $T$ is the temperature can be given as [3]:

$$
B(T)=C(T)+D
$$

In (17), $C$ is the slope of the plot and $D$ is the $x$ intercept. Using (17) in (16), we have the equation [3]:

$$
\begin{aligned}
& \sqrt{\xi}=A(M C)+C(T)+D \\
& M C=\frac{\sqrt{\xi}-C(T)-D}{A}
\end{aligned}
$$

Thus (19) is the bulk density independent, temperature compensated calibration equation which can be used to calculate the moisture content. If $\mathrm{N}$ represents the number of samples and $\Delta \mathrm{Mi}$ represents the difference in actual value and predicted value of moisture content for the ith sample, the standard error of calibration (SEC) can be calculated as [3]:

$$
S E C=\sqrt{\frac{1}{N-1} \sum_{i=1}^{W}(\Delta M i)^{2}}
$$

\section{CONCLUSION}

The review of microwave based measurement technique shows clear advantage of using horn antennas over patch antennas due to the requirement of high directivity though being bulky. The calibration based on only attenuation needs to be temperature and bulk density compensated. The two parameter ratio method of calibration is simple and achieves bulk density independence. However, it is more suitable for transmission techniques. The density independent calibration method is the most suitable method. This method is related to dielectric properties of the material under test and hence can be used regardless of the measurement techniques. Along with bulk density independence, it is easily temperature compensated and can also be used for calculating bulk density thus eliminating the need of a separate device for bulk density measurement.

\section{REFERENCES}

[1] ASAE S352.2 Moisture Measurement-Unground grain and seeds, ASAE, St. Joseph, MI, ASAE, 1995, ASAE Standards 1995

[2] T. J. Kelleners, D. A. Robinson, P. J. Shouse, J. E. Ayars, and T. H. Skaggs, Frequency Dependence of the Complex Permittivity and Its Impact on Dielectric Sensor Calibration in Soils, Soil Sci. Soc. Am. J., Vol. 69, pp 67-76, January- February2005

[3] Samir Trabelsi, Andrzej W. Krazsewski and Stuart O. Nelson, New Density-Independent Calibration Function for Microwave Sensing of Moisture Content in Particulate Materials, IEEE, IEEE Transactions On Instrumentation And Measurement, Vol. 47, No. 3, June 199, pp 613-622

[4] Samir Trabelsi and Stuart O. Nelson, Microwave Moisture Meter for Granular and Particulate Materials, Instrumentation and Measurement Technology Conference (I2MTC), 2010 IEEE, pp 1304-1308

[5] El Sabbagh, Richard B. Russell, Ramahi, O.M. ; Trabelsi, S. ; Nelson, S.O. ; Khan, L, Use of Microstrip Patch Antennas in Grain and Pulverized Materials Permittivity Measurement, Antennas and Propagation Society International Symposium, 2003, IEEE, Vol 4, pp 42-45

[6] Stuart O. Nelson, Samir Trabelsi, and Andrzej W. Kraszewski, Principles of Microwave Moisture Measurement in Grain, Instrumentation and Measurement Technology Conference, 2002. IMTC/2002. Proceedings of the 19th IEEE, Vol 1, pp 99-102

[7] A.W. Kraszewski, S. Trabelsi, S.O. Nelson, Simple Grain and Moisture Content Determination from Microwave Measurements, Transactions of the ASAE, 1998 American Society of Agricultural Engineers, Vol. 41, pp 129-134

[8] Ki-Bok Kim, Jong-Heon Kim, Seung Seok Lee, and Sang Ha Noh, Measurement of Grain Moisture Content Using Microwave Attenuation at $10.5 \mathrm{GHz}$ and Moisture Density, IEEE Transactions On Instrumentation And Measurement, Vol. 51, No. 1, February 2002

[9] Yangjun Zhang and Seichi Okamura, A Density-Independent Method For High Moisture Content Measurement using a Microstrip Transmission Line, International Microwave Power Institute, Journal of Microwave Power \& Electromagnetic Energy Vol. 40, No.2, 2006

[10] A.R. von Hippel, Dielectrics and Waves. New York: Wiley, 1954, p. 26 